\title{
Anti-Coarsening and Complex Dynamics of Step Bunches on Vicinal Surfaces during Sublimation
}

\author{
Marian Ivanov, ${ }^{1}$ Vladislav Popkov, ${ }^{2,3}$ and Joachim $\mathrm{Krug}^{1}$ \\ ${ }^{1}$ Institut für Theoretische Physik, Universität zu Köln, 50937 Köln, Germany \\ ${ }^{2}$ Interdisziplinäres Zentrum für komplexe Systeme, \\ Rheinische Friedrich-Wilhelms-Universität Bonn, 53117 Bonn, Germany \\ ${ }^{3}$ Dipartimento di Fisica Teorica "E. R. Caianiello", \\ Università degli Studi di Salerno, Salerno, Italy
}

(Dated: May 25, 2022)

\begin{abstract}
A sublimating vicinal crystal surface can undergo a step bunching instability when the attachment-detachment kinetics is asymmetric, in the sense of a normal Ehrlich-Schwoebel effect. Here we investigate this instability in a model that takes into account the subtle interplay between sublimation and step-step interactions, which breaks the volume-conserving character of the dynamics assumed in previous work. On the basis of a systematically derived continuum equation for the surface profile, we argue that the non-conservative terms pose a limitation on the size of emerging step bunches. This conclusion is supported by extensive simulations of the discrete step dynamics, which show breakup of large bunches into smaller ones as well as arrested coarsening and periodic oscillations between states with different numbers of bunches.
\end{abstract}

PACS numbers: 05.70.Np, 68.35.Ct, 81.16.Rf 


\section{INTRODUCTION}

We consider a vicinal crystal surface of parallel oriented steps in contact with the vapor phase of the same material. By varying the sample temperature the crystal can start to sublimate or grow in the step flow mode [1 4]. In the presence of an asymmetry in the kinetics at the steps bordering a terrace, perturbations in the step flow may grow such that the step profile undergoes a step bunching instability. There are two questions of general interest. First, what are the physical conditions required for such an instability? And second, how does the surface morphology evolve once the instability has appeared?

In recent years many groups have addressed these questions within the framework of the standard model first introduced by Burton, Cabrera and Frank [5] (BCF). In the absence of additional effects such as electromigration [6, 7] or strain [8], the basic stability scenario is simple: Preferential attachment to ascending steps (a normal Ehrlich-Schwoebel effect) leads to step bunching during sublimation, while preferential attachment to descending steps (an inverse Ehrlich-Schwoebel effect) implies step bunching during growth [9 14]. The symmetry between growth and sublimation suggested by this result is however not complete [15, 16]: Whereas the deposition flux in a growth experiment is an externally controlled parameter that is independent of the surface morphology, the sublimation flux is driven by the surface free energy and hence depends on the curvature of the surface. As a consequence, the time evolution of the surface profile conserves the volume of the film (apart from a constant rate of increase) in the case of growth but not in the case of sublimation [17].

In the present paper we explore the consequences of the non-conservative nature of the sublimation dynamics for the linear and nonlinear evolution of step bunches. We base our treatment on the BCF model in the quasistatic limit including sublimation, the EhrlichSchwoebel effect and step-step interactions. In previous work we have developed a detailed continuum description of step bunching in this system, in which non-conservative terms arising from the interplay between sublimation and capillarity were however neglected [18, 19]. Here these terms are explicitly included and their effect is studied both in the discrete dynamics of individual steps and on the continuum level.

Although the coefficients of the non-conserved terms are usually small under physically realistic conditions, they turn out to have dramatic consequences. Most importantly, whereas step bunches in conserved systems typically coarsen indefinitely, here we find that coarsening 
is arrested when a maximal bunch size has been reached. Conversely, if the system of steps is started in an initial condition representing a single large bunch, anti-coarsening involving the breakup of the initial bunch into several small bunches is observed. More complex scenarios in which the number of bunches in the system varies periodically in time are also possible.

The article is organized as follows. In the next section we briefly introduce the BCF-model which forms the starting point of our work. In Section [II] the discrete equations of motion for the steps are presented and the results of a linear stability analysis are described. In Section [V] we derive a continuum evolution equation along the lines of [18, 20] and provide a partial analysis which suggests the existence of an upper bound on the size of step bunches in the non-conserved case. Section $\nabla$ is devoted to the numerical exploration of the various scenarios of nonlinear evolution in the discrete step dynamics, and some conclusions are presented in Section VI. Details of the analytic calculations are collected in the Appendices.

\section{MODEL}

On the mesoscopic scale the surface can be reduced to a one-dimensional train of steps. The BCF model is based on a non-conserved diffusion equation for the concentration profile $n_{i}(x, t)$ on the $i$ 'th terrace (see Fig.1), which reads

$$
\frac{\partial n_{i}(x, t)}{\partial t}=D_{s} \frac{\partial^{2} n_{i}(x, t)}{\partial x^{2}}-\frac{n_{i}(x, t)}{\tau}+F .
$$

The terms on the right hand side correspond to the three processes sketched in Fig. 1. The first one is a diffusion term with surface diffusion coefficient $D_{s}$, the second term includes the losses of adatoms during sublimation with desorption rate $1 / \tau$, and the last one describes the gain of adatoms from the surrounding gas phase with deposition rate $F$. Together diffusion and desorption give rise to the diffusion length $l_{D}=\sqrt{D_{s} \tau}$, defined as the distance an adatom travels before it desorbs (in the absence of other processes).

Solving (11) in the quasistatic limit $\partial n_{i} / \partial t=0$ one can find the general solution. The constants of integration are specified through the following boundary conditions. We consider a terrace of width $l$ confined between two steps at the positions $x= \pm l / 2$. The condition of mass conservation on both bounding steps defines the system of differential equations

$$
\begin{aligned}
D_{s} \frac{\partial n(x)}{\partial x} & =+k_{-}\left[n(x)-n_{e q}(x)\right], & \text { at } x & =-\frac{l}{2}, \\
D_{s} \frac{\partial n(x)}{\partial x} & =-k_{+}\left[n(x)-n_{e q}(x)\right], & \text { at } x & =+\frac{l}{2} .
\end{aligned}
$$




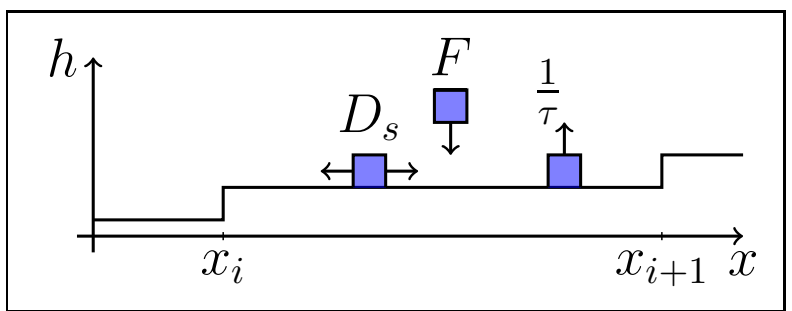

FIG. 1: (Color online) Sketch of the elementary processes in the Burton-Cabrera-Frank model

On the left hand sides the adatom fluxes from the terrace toward the steps appear, which are compensated by the attachment and detachment of adatoms that are caused by the difference between the actual concentration at the step relative to the equilibrium concentrations.

The system (2) contains two additional effects. First, the proportionality constants $k_{-}$and $k_{+}$are the attachment/detachment kinetic coefficients, where index the $+(-)$denotes the coefficient corresponding to attachment/detachment from below (above) the step. In general the $k_{ \pm}$are unequal. The case $k_{+}>k_{-}$corresponds to the so called Ehrlich-Schwoebel effect [9] while in the converse case we speak about an inverse Ehrlich-Schwoebel effect [12 14]. Analogous to the diffusion length we define the kinetic lengths $l_{ \pm}=D_{s} / k_{ \pm}[2]$ and further their dimensionless versions $l_{ \pm} / l_{D}=l^{ \pm}$(note the different placement of the indices \pm ).

The second effect included in the boundary conditions (2) are the (repulsive) step-step interactions. The expression for the equilibrium concentration is given by the usual grand canonical formula, which we use in the first order approximation

$$
n_{e q}\left(x_{i}\right) \approx n_{e q}^{0}\left(1+\mu_{i} / k_{B} T\right)
$$

The chemical potential $\mu_{i}$ at the $i$ th step depends on the widths $l_{i}=x_{i}-x_{i-1}$ of the neighboring terraces according to the law

$$
\frac{\mu_{i}}{k_{B} T}=-g\left[\frac{l^{3}}{\left(l_{i}\right)^{3}}-\frac{l^{3}}{\left(l_{i-1}\right)^{3}}\right] \equiv g \nu_{i},
$$

which was first derived theoretically for entropic repulsion [21]. The ubiquity of repulsive step-step interactions is well confirmed by experiments, which also show that the dominant contribution to the amplitude $g$ arises from elastic interactions [1, 22].

In order to render $g$ dimensionless we have rescaled the terrace widths in (44) by the mean terrace width $l$. Note that our definition of $g$ differs from the conventional notation, where the strength of step interactions is quantified by the coefficient of the cubic term in the 


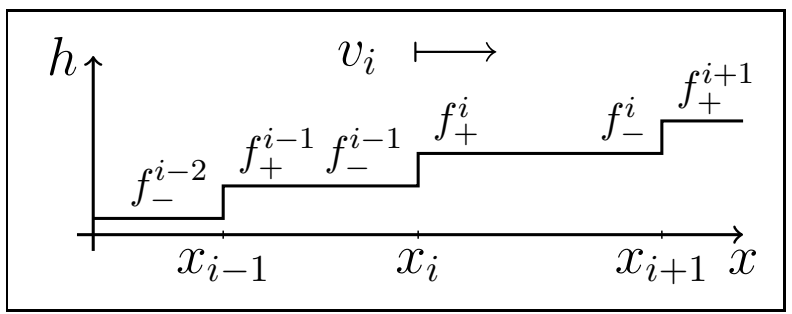

FIG. 2: Sketch of the contributions to the velocity of the $i$-th step.

expansion of the surface free energy in the surface miscut $\theta$, the angle formed by the vicinal surface relative to the high symmetry orientation [1]. Denoting the latter coefficient by $\tilde{g}$, the relation between the two reads [23]

$$
g=\frac{2 \tilde{g} \Omega}{k_{B} T}|\tan \theta|^{3}
$$

where $\Omega$ denotes the atomic area. To give an impression of the order of magnitude of $g$, for the $\operatorname{Si}(111)$ surface at $900^{\circ}$ the estimate $\tilde{g} \approx 0.05 \mathrm{eV} / \AA^{2}[1]$ yields $g \approx 10|\tan \theta|^{3} \approx 5 \times 10^{-5}$ for a typical miscut of $1^{\circ}$.

Together Eqs.(1,2,3,4) specify the boundary value problem for the $n_{i}(x)$. Having computed the concentration profiles we can use Fick's first law to find the mass fluxes from below $\left(f_{+}^{i-1}\right)$ and above $\left(f_{-}^{i}\right)$ the $i$-th step, which sum to give its velocity $v_{i}=\frac{d x_{i}}{d t}=f_{-}^{i}+f_{+}^{i-1}$ (see Fig. 2).

\section{DISCRETE VIEW}

\section{A. Equations of step motion}

In Appendix A1 we derive the concentration profile $n_{i}(x)$ for the case of sublimation $(F=0)$ with step-step repulsion and the Ehrlich-Schwoebel effect in the quasistatic limit, see Eq. (A1) and Eq. (A4), which yields

$$
\begin{aligned}
n_{i}(x) & =n_{e q}^{0}\left[\frac{\left(l^{+}-1\right)\left(1+\frac{\mu_{i}}{k T}\right) e^{-\frac{l_{i}}{2 l_{D}}}+\left(l^{-}+1\right)\left(1+\frac{\mu_{i+1}}{k T}\right) e^{\frac{l_{i}}{2 l_{D}}}}{\left(l^{+} l^{-}+1\right) \sinh \frac{l_{i}}{l_{D}}+\left(l^{-}+l^{+}\right) \cosh \frac{l_{i}}{l_{D}}}\right] \frac{e^{\frac{x}{l_{D}}}}{2}+ \\
& +n_{e q}^{0}\left[\frac{\left(l^{-}-1\right)\left(1+\frac{\mu_{i}}{k T}\right) e^{-\frac{l_{i}}{2 l_{D}}}+\left(l^{+}+1\right)\left(1+\frac{\mu_{i+1}}{k T}\right) e^{\frac{l_{i}}{2 l_{D}}}}{\left(l^{+} l^{-}+1\right) \sinh \frac{l_{i}}{l_{D}}+\left(l^{-}+l^{+}\right) \cosh \frac{l_{i}}{l_{D}}}\right] \frac{e^{-\frac{x}{l_{D}}}}{2} .
\end{aligned}
$$


From (5) we find the following discrete equations of step motion (see Appendix A 2)

$$
\begin{aligned}
\frac{d x_{i}}{d t} & =\frac{\Omega D_{s} n_{e q}^{0}}{l_{D}}\left[\frac{\left(1+\frac{\mu_{i}}{k T}\right)\left(l^{-} \sinh \frac{l_{i-1}}{l_{D}}+\cosh \frac{l_{i-1}}{l_{D}}\right)-\left(1+\frac{\mu_{i-1}}{k T}\right)}{\left(l^{+} l^{-}+1\right) \sinh \frac{l_{i-1}}{l_{D}}+\left(l^{-}+l^{+}\right) \cosh \frac{l_{i-1}}{l_{D}}}\right]+ \\
& +\frac{\Omega D_{s} n_{e q}^{0}}{l_{D}}\left[\frac{\left(1+\frac{\mu_{i}}{k T}\right)\left(l^{+} \sinh \frac{l_{i}}{l_{D}}+\cosh \frac{l_{i}}{l_{D}}\right)-\left(1+\frac{\mu_{i+1}}{k T}\right)}{\left(l^{+} l^{-}+1\right) \sinh \frac{l_{i}}{l_{D}}+\left(l^{-}+l^{+}\right) \cosh \frac{l_{i}}{l_{D}}}\right]
\end{aligned}
$$

The step velocities (6) include all three length scales $l_{D}, l_{ \pm}, l$ and the functional dependence is quite complicated. From now on we therefore consider the case of attachment/detachment limited kinetics $\left(l_{D} \gg l_{ \pm} \gg l\right)$, which is commonly assumed for the $\operatorname{Si}(111)$ surface [23]. In this limit Eq. (6) can be reduced to the following form (see Appendix A4):

$$
\frac{d x_{i}}{d t}=\left(1+g \nu_{i}\right) R_{e}\left(\frac{1+b}{2} l_{i-1}+\frac{1-b}{2} l_{i}\right)+U R_{e}\left(2 \nu_{i}-\nu_{i-1}-\nu_{i+i}\right),
$$

with the abbreviations

$$
\begin{aligned}
b & =\frac{k_{+}-k_{-}}{k_{-}+k_{+}}=\frac{l_{-}-l_{+}}{l_{-}+l_{+}}, \\
R_{e} & =\frac{\Omega n_{e q}^{0}}{\tau} \\
U & =g \tau \frac{k_{-} k_{+}}{k_{-}+k_{+}}=\frac{g l_{D}^{2}}{l_{-}+l_{+}} .
\end{aligned}
$$

Here $R_{e}$ represents the constant desorption rate of a homogeneous step train, where $d x_{i} / d t=$ $R_{e} l, b$ is a dimensionless measure for the strength of the Ehrlich-Schwoebel effect and $U$ describes the strength of the relaxation due to the step-step repulsion. Note that $U$ has the dimension of a length.

In previous work on step bunching during sublimation, where the instability is induced either by the Ehrlich-Schwoebel effect or by electromigration, the factor $1+g \nu_{i}$ of the first term on the right hand side of (17) was tacitly replaced by unity [18, 19, 23, 24]. The approximation $1+g \nu_{i} \approx 1$ may seem plausible because, as we have seen, $g \ll 1$ under typical experimental conditions. However, it is clear from the structure of (7) that the presence of this factor changes the nature of the problem in a qualitative way: Considering periodic boundaries for the step train of $M$ steps, so that $x_{M+1}=x_{1}+M l$, and taking the sum of (7) over one period one obtains the configuration-independent constant $M l$ on the right hand size only when the term $g \nu_{i}$ (referred to in the following as the $g$-term) is neglected. The full system (7) is fundamentally non-conservative. 


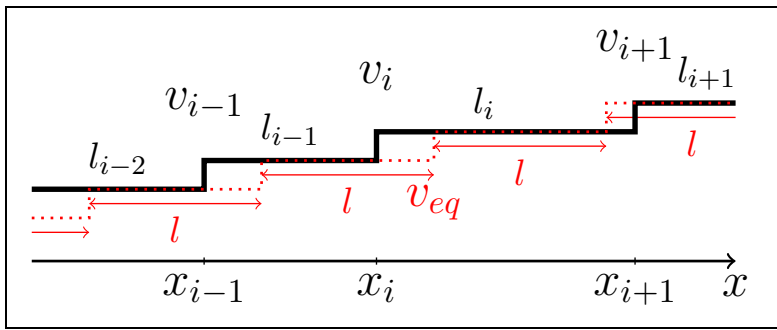

FIG. 3: (Color online) Illustration of the linear stability analysis. The dotted line shows a homogeneous step train with constant terrace width $l$ and constant step velocity $v_{e q}$, the full line shows a perturbed step train.

On the other hand, the step dynamics is exactly conservative for the case of pure growth $(F>0, \tau \rightarrow \infty)$ in the same limit in which (7) was derived. In the case of growth we obtain

$$
\frac{d x_{i}}{d t} \approx-F \Omega\left(\frac{1+b}{2} l_{i-1}+\frac{1-b}{2} l_{i}\right)+\tilde{U}\left(2 \nu_{i}-\nu_{i-1}-\nu_{i+i}\right)
$$

which is precisely the conservative version of (7) with the sublimation rate $R_{e}$ replaced by the (negative) growth rate $F \Omega$ and with $\tilde{U}=\left(g \Omega n_{e q}^{0} k_{-} k_{+}\right) /\left(k_{-}+k_{+}\right)$. This implies a basic asymmetry between sublimation and growth, the consequences of which will be explored in the following. To this end we will consider $U$ and $g$ in (7) as independent parameters, in spite of the proportionality between $U$ and $g$. The conserved model (9) is thus included in (17) as the limiting case $g=0, U>0$.

\section{B. Linear stability analysis}

The instability form of a step train is step bunching and in this section we look for the linear instability condition as a function of the control parameters. Let us consider the regular situation of equidistant terrace widths $l$ and steps moving with a constant velocity $v_{e q}=f_{-}(l)+f_{+}(l)$, see Fig. 3. Now, we disturb the positions of the steps by a small time dependent perturbation $\varepsilon_{n}(t)$ :

$$
x_{n}(t)=n l+v_{e q} t+\varepsilon_{n}(t) .
$$

Through substitution of $\varepsilon_{n}(t)$ by the Fourier expression $\varepsilon_{0} e^{i k n+\omega(k) t}$ we derive a dispersion relation $\omega(k)$. For instability the real part of $\omega(k)$ has to be positive, i.e.

$$
\operatorname{Re}[\omega(k)] \approx A_{2} k^{2}+A_{4} k^{4}
$$




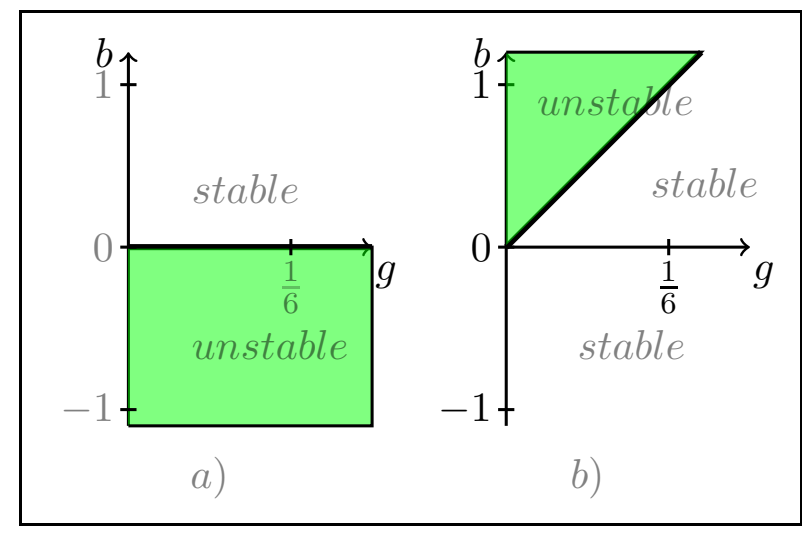

FIG. 4: (Color online) Stability diagram in the $(g, b)$-plane for a) growth and b) sublimation.

with $A_{2}>0$. In Appendix A3, Eq. (A8), we find:

$$
R e[\omega(k)]=\frac{\Omega D_{s} n_{e q}^{0}}{l_{D}^{2}}\left[\frac{\left(k_{+}-k_{-}\right)}{2\left(k_{+}+k_{-}\right)}-3 g\right] k^{2}-3 g \frac{\Omega D_{s} n_{e q}^{0}}{l} \frac{k_{-} k_{+}}{k_{-}+k_{+}} k^{4} .
$$

For the case of sublimation in the limit of long wavelengths (small $k$ ) the instability condition is $b>6 g$. This means that the existence of an Ehrlich-Schwoebel effect alone $(b>0)$ is not sufficient to cause an instability: Due to the step-step interactions there is a lower limit $6 g$ on the strength of the kinetic asymmetry which has to be overcome to cause step bunching during sublimation. This effect was previously reported by Fok, Rosales and Margetis [16] in a more general setting. On the other hand for the case of growth we have the usual linear instability condition of $b<0$, which corresponds to an inverse Ehrlich-Schwoebel effect of arbitrary strengh. The resulting asymmetry between sublimation and growth on the level of linear stability analysis is illustrated in Fig. 4.

\section{CONTINUUM DESCRIPTION}

The continuum evolution equation corresponding to the discrete dynamics (7) has the form

$$
\frac{\partial h}{\partial t}+\frac{\partial}{\partial x}\left(-\frac{3 g}{2}\left(m^{2}\right)-\frac{b}{2 m}-\frac{1}{6 m^{3}} \frac{\partial m}{\partial x}+\frac{3 U}{2 m} \frac{\partial^{2}\left(m^{2}\right)}{\partial x^{2}}\right)=-1+\frac{3 g b}{2 m}\left(\frac{\partial m}{\partial x}\right)^{2}
$$

where $h(x, t)$ is the surface profile, $m(x, t)=\partial h / \partial x>0$ is the slope, and primes denote the derivatives with respect to $x$, e.g. $m^{\prime}=\partial m / \partial x$. Here and below we set the step height and the average step-step distance $l$ to 1 , and rescale time such that the mean sublimation rate $R_{e}=1$. The details of the derivation can be found in Appendix B. 
For $g=0$, the equation (10) reduces to the one studied in [18, 19]. In that case the transformation to a moving frame $h \rightarrow h+t$ removes the constant mean sublimation rate on the right hand side and gives the equation the form of a conservation law

$$
\frac{\partial h}{\partial t}+\frac{\partial J}{\partial x}=0
$$

with a current $J$ defined by the terms inside the large parentheses on the left hand side of (10). For $g>0$ we obtain an additional contribution $(3 g / 2) m^{2}$ to the current, and the manifestly non-conservative term $(3 \mathrm{gb} / 2 \mathrm{~m})\left(\mathrm{m}^{\prime}\right)^{2}$ appears on the right-hand side of (10). In the following we discuss separately the effects of the conserved and non-conserved contributions that are present when $g>0$.

\section{A. Mechanical analog for symmetric stationary bunches}

We first analyze the effect of the conservative term $(3 \mathrm{~g} / 2) \mathrm{m}^{2}$ on the left hand side of (10). Along the lines of [18], we consider a stationary solution of (11) with the current given by

$$
J=-\frac{3 g}{2}\left(m^{2}\right)-\frac{b}{2 m}-\frac{m^{\prime}}{6 m^{3}}+\frac{3 U}{2 m}\left(m^{2}\right)^{\prime \prime} .
$$

For a stationary bunch $J \equiv-J_{0}<0$. Furthermore we neglect the third term on the right hand side of (12), which breaks the reflection symmetry $(x \rightarrow-x$ and $m \rightarrow-m)$. Setting $u=m^{2}(m>0)$ then yields

$$
J_{0}=\frac{3 g}{2} u+\frac{b}{2} u^{-1 / 2}-\frac{3 U u^{\prime \prime}}{2} u^{-1 / 2}
$$

or

$$
\frac{3 U}{2} u^{\prime \prime}=-J_{0} u^{1 / 2}+\frac{3 g}{2} u^{3 / 2}+\frac{b}{2}=-\frac{d V_{g}(u)}{d u} .
$$

Equation (13) can be interpreted as Newton's second law describing a particle coordinate $u$ moving in time $x$. In this picture a symmetric bunch of width $L$ represents a trajectory of a classical particle moving once back and forth in a potential $V_{g}(u)$ between the boundary values $u=0$ and $u=u_{\max }$ in a time $L$. Up to a constant, the potential $V_{g}(u)$ can be found through integration of (13)),

$$
V_{g}(u)=\frac{2 J_{0}}{3} u^{3 / 2}-\frac{3 g}{5} u^{5 / 2}-\frac{b}{2} u=V_{0}(u)-\frac{3 g}{5} u^{5 / 2} .
$$


The function (14) contains an additional term compared to the potential $V_{0}(u)$ considered in [18]. This term causes a maximum of $V_{g}(u)$ to appear at some $u^{*}$, whereas $V_{0}(u)$ grows monotonically for large $u$. This has the following consequence. Let us consider a bunch of $M$ steps. For a given set of parameters $b, g$ and $U$ this bunch solution corresponds to a particle trajectory of total energy $E$ and a certain maximal slope $m_{\max }$ with $u_{\max }=m_{\max }^{2}<u^{*}$. Now, let us increase the bunch width $L$ by adding more and more steps, which increases the energy $E$ and the value of $u_{\max }$. As $u_{\max } \rightarrow u^{*}$ and $E \rightarrow V_{g}\left(u^{*}\right)$, the oscillation period $L$ of the particle diverges, which implies that the maximum slope $m_{\max }$ cannot increase beyond $\sqrt{u^{*}}$. This is in contrast to the case $g=0$, where the maximum slope scales with the number of steps as $m_{\max } \sim M^{2 / 3}$ and with the bunch width as $m_{\max } \sim L^{2}$ [18]. Even on the rather crude level of the stationary bunch approximation used in [18], the presence of the $g$-term in (12) is seen to have a pronounced effect, in that it prevents the unbounded steepening of the bunch profile. In the next subsection we shall see that, when bunch motion is taken into account, the non-conserved nature of the dynamics also prevents the wavelength of the bunch from increasing indefinitely.

\section{B. Moving bunches}

In fact step bunches are not stationary, but move both in the horizontal direction (with speed $V_{\|}$) and in the vertical direction (with speed $V_{\perp}$ ) [19]. A periodic array of moving bunches is obtained from (10) using the travelling wave ansatz

$$
h(x, t)=h\left(x-V_{\|} t\right)+V_{\perp} t-t,
$$

which yields the ordinary differential equation

$$
-V_{\|} h^{\prime}+V_{\perp}+\left(-\frac{3 g}{2}\left(m^{2}\right)-\frac{b}{2 m}-\frac{m^{\prime}}{6 m^{3}}+\frac{3 U}{2 m}\left(m^{2}\right)^{\prime \prime}\right)^{\prime}=\frac{3 g b}{2} \frac{\left(m^{\prime}\right)^{2}}{m} .
$$

Here primes denote derivatives with respect to co-moving space coordinate $\xi=x-V_{\|} t$ and $m(\xi)=d h / d \xi$. We look for periodic solutions $h(\xi)=h(\xi+M)$. Integrating (15) from 0 till $M$, and dividing by $M$ we obtain

$$
V_{\|}-V_{\perp}=g \frac{3 b}{2 M} \int_{0}^{M} \frac{\left(m^{\prime}\right)^{2}}{m} d \xi
$$




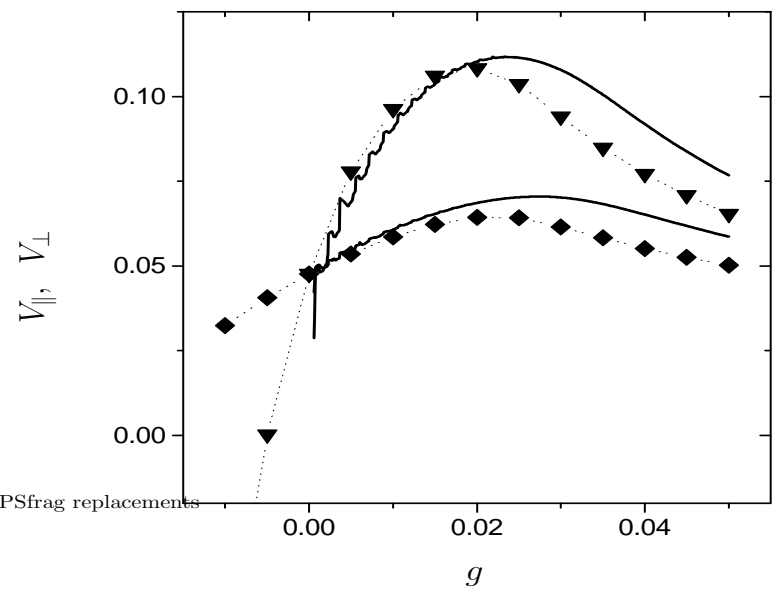

FIG. 5: Horizontal and vertical bunch speeds $V_{\|}, V_{\perp}$ versus $g$ for the discrete model (diamonds and triangles, respectively), and for the continuous model (thick lines). Parameters are $b=0.7, U=$ $0.5, M=40$.

which implies $V_{\|}=V_{\perp}$ in the conserved case [19]. In a linear order approximation in $g$, the integral on the right-hand side can be estimated by its value at $g=0$. The latter can be computed analytically using the theory developed in [19], with the result

$$
\int_{0}^{M} \frac{\left(m^{\prime}\right)^{2}}{m} d \xi=\frac{1}{36 U m_{\min }^{2}} \approx \frac{(3 b)^{2}}{36 U}=\frac{b^{2}}{4 U} M^{2} .
$$

Here $m_{\text {min }}$ denotes the minimal value of the slope along the profile. This leads to the prediction

$$
V_{\perp}(g)-V_{\|}(g)=g \frac{3 b^{3}}{8 U} M \text { for small } g .
$$

This result is in reasonable agreement with the direct measurement of $V_{\|}$and $V_{\perp}$, performed for the discrete model (Figures 5 and [6). From Fig. 5 we estimate $V_{\perp}(g)-V_{\|}(g) \approx \alpha g$ for small $g$ with $\alpha \approx 8$, while the direct calculation from (16) using the parameters $b=0.7, U=$ $0.5, M=40$, gives $\frac{3 b^{3}}{8 U} M=\alpha=10.3$. From Fig. 6] we estimate $\alpha \approx 3$ while the theoretical prediction gives $\alpha \approx 3.75$.

The result (16) is quite surprising: It tells us that the difference $V_{\perp}(g)-V_{\|}(g)$ acquires a singularity at $g=0$ in the limit $M \rightarrow \infty$, where continuum theory should become accurate. To avoid this unphysical singularity, we must assume the existence of an upper bound on $M$ (and hence, on the wavelength of moving bunch solutions) for small but positive values of $g$. Indeed, in the discrete simulations to be reported in the next section, we will see that the 


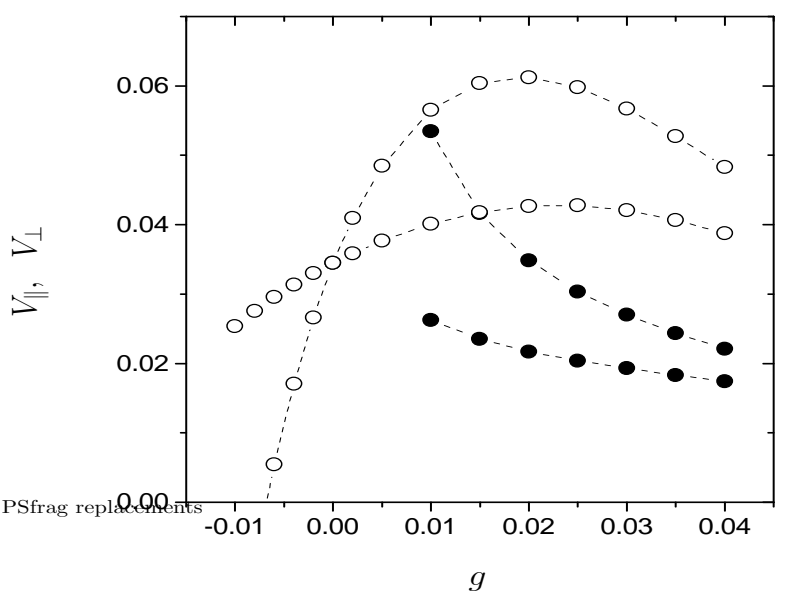

FIG. 6: Horizontal and vertical bunch speeds as functions of $g$ for the parameters $b=0.5, U=0.5$ and $M=40$ (open circles) and $M=80$ (filled circles) obtained from simulations of the discrete model. Results for $M=80$ are reported only for large $g$. In the physical region $g>0, V_{\perp}>V_{\|}$, which corresponds to step trajectories in the comoving system of coordinates going downwards (compare to Figs.7 and 8). For large $g$, the absolute values and the difference $V_{\perp}=V_{\|}$are decreasing functions of $M$. For small $g, V_{\perp}-V_{\|} \approx \alpha g$ where $\alpha \approx 3$. From Eq.(16) we get the theoretical estimate $\alpha=\frac{3 b^{3}}{8 U} M=3.75$

coarsening is arrested at a certain maximum wavelength $M_{\text {crit }}(g)$. On the other hand, since in the conservative case $(g=0)$ the vertical and horizontal excess speed of the bunch are equal, $V_{\perp}=V_{\|}$, we expect from (16) that $\lim _{g \rightarrow 0} g M_{\text {crit }}(g)=0$. In other words, we deduce that for small $g$ the critical bunch size $M_{\text {crit }}$ must grow more slowly than $1 / g$. Note also that in the conservative case the values of the excess velocities decrease with the bunch size $M$ as $V_{\perp}=V_{\|} \approx 3 b / M[19]$. We observe a similar tendency (decrease of $V_{\perp}$ and $V_{\|}$with increasing $M$ ) for large enough $g$, compare the upper and the lower curves in Fig, 6 ,

\section{NUMERICAL SIMULATIONS OF THE DISCRETE EQUATIONS}

We simulated the coupled system (7) of $M$ non-linear ordinary differential equations of first order with four independent parameters: $M$ is the number of steps, $g$ is the parameter describing the non-conserving effects, $b$ is the parameter describing the Ehrlich-Schwoebel asymmetry and $U$ is the relaxation parameter due to the step-step interactions. We studied the following region of the parameter space: $b \in[0,1], U \in[0,1], g \in[0,1]$ and $M<100$. 
Note that, according to the definition of $U$ in Eq.(8) $)$, the dimensionless ratio $g l / U=l\left(l_{-}+\right.$ $\left.l_{+}\right) / l_{D}^{2}$ should be small compared to unity in order to be consistent with the assumption $l_{D} \gg l_{ \pm} \gg l$ under which the model (7) was derived. Since our main interest here is in exploring the qualitative consequences of the non-conserved dynamics induced by the $g$-term, rather than in a realistic description of a particular physical system, we will not always adhere to this restriction.

For the numerical integration we used an odeint-type procedure [25] with periodic boundary conditions. The height of a single step and the average terrace width $l$ are normalized to unity. The time scale is normalized by $R_{e}$ and we measured the time of integration in time units (t.u.). We started with two qualitatively different initial conditions: first, an initial 'shock' composed of densely packed steps and a very large terrace, and second, a randomly disturbed equidistant step train, where the relative amplitude of initial fluctuations is chosen to be either small (0.01) or large (0.5).

We define a bunch as a region where the widths $l_{i}$ of consecutive terraces are smaller than one. Figure $7 \mathrm{a})$ shows a step train profile in the linear instability regime $(b>6 g)$. As useful quantitative measures of the bunch geometry we define the maximal slope $m_{\max } \equiv \max _{i}\left\{m_{i}\right\}$ and the minimal (=maximally negative) curvature $\kappa_{\min } \equiv \min _{i}\left\{\kappa_{i}\right\}$, where

$$
m_{i}=\frac{1}{l_{i}}, \quad \kappa_{i}=-8 \frac{l_{i+1}-l_{i}}{\left(l_{i+1}+l_{i}\right)^{3}} .
$$

We calculate and plot the positions of the steps in a co-moving coordinate system defined by $\tilde{x}_{i}(t)=x_{i}(t)-l t$, where $l$ is the average velocity in the conserved limit $(g=0)$. Because of the additional nonconservative terms, there is a lateral shift after every period, see Fig. 7b) and also Fig. 8 8 )

For simulations with $b \lesssim 6 g$ an initial step bunch dissolves and approaches the configuration of equidistant steps. On the other hand for $b>6 g$ and increasing $g / U$, the time evolution of the step train switches unexpectedly to anti-coarsening or arrested coarsening regimes, depending on the initial conditions. In the following we describe these two scenarios in more detail. 

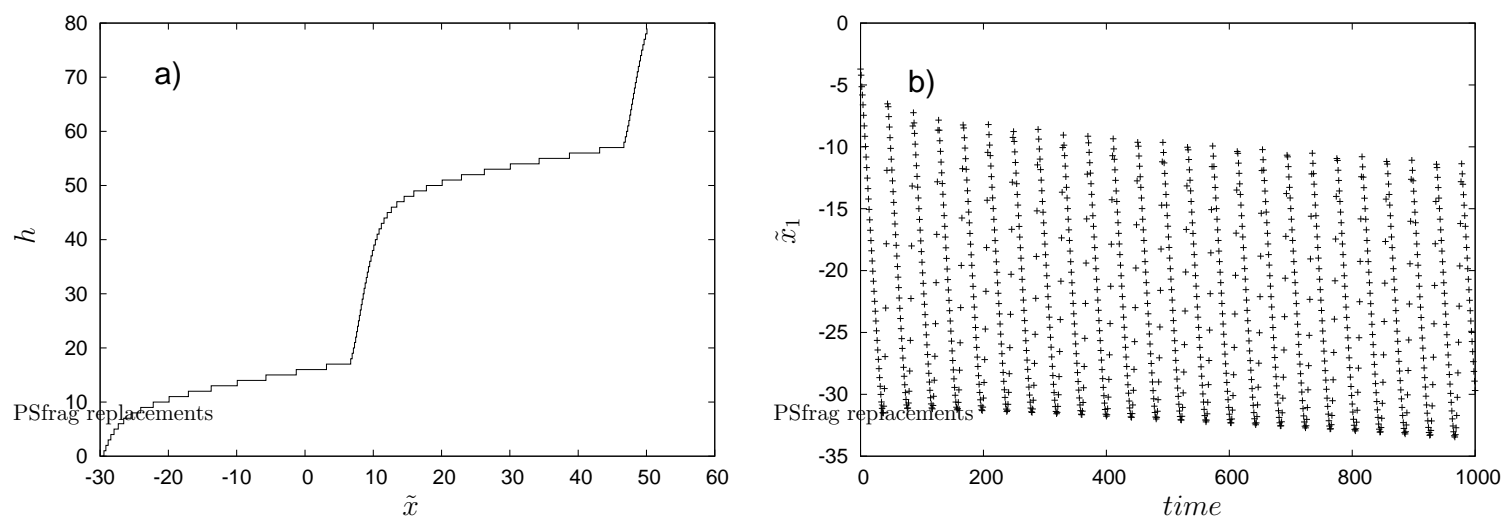

FIG. 7: Simulation results for $M=40, b=0.1, g=0.001, U=0.004$, starting with an initial shock and periodic boundary conditions. a) Typical step train profile after 1000 t.u.; b) Typical time evolution of one of the steps in co-moving coordinates.

\section{A. Anti-Coarsening}

The first surprising result we observed is the splitting of a bunch into two or more bunches after starting with an initial shock of steps. Let us consider the example in Fig. 8. The system consists of 80 steps with parameters $b=0.7, g=0.05$ and $U=0.05$. Figure 8 a) shows the time evolution of the position $x_{1}$ of one of the steps. There is a constant shift until the bunch splits, and then the shift increases, which corresponds to a much faster moving step. In Fig. 8b) we plot the positions of all steps around the time of the spliting, where we can see the emergence of an additional, very small but growing bunch. The splitting event is followed by another one and so forth until there are five almost equally sized bunches at 8000 t.u., see the surface height profiles in 8 c). In Fig. 8d) we show the time evolution of the minimal curvature, the maximal slope and the number of bunches. In the splitting region the minimal curvature is seen to show a strong signature with an abrupt jump followed by relaxation to a smaller value, whereas the change in the maximal slope is rather small. The splitting of bunches in this system is an example of anti-coarsening, where a large structure breaks up into multiple small structures. 

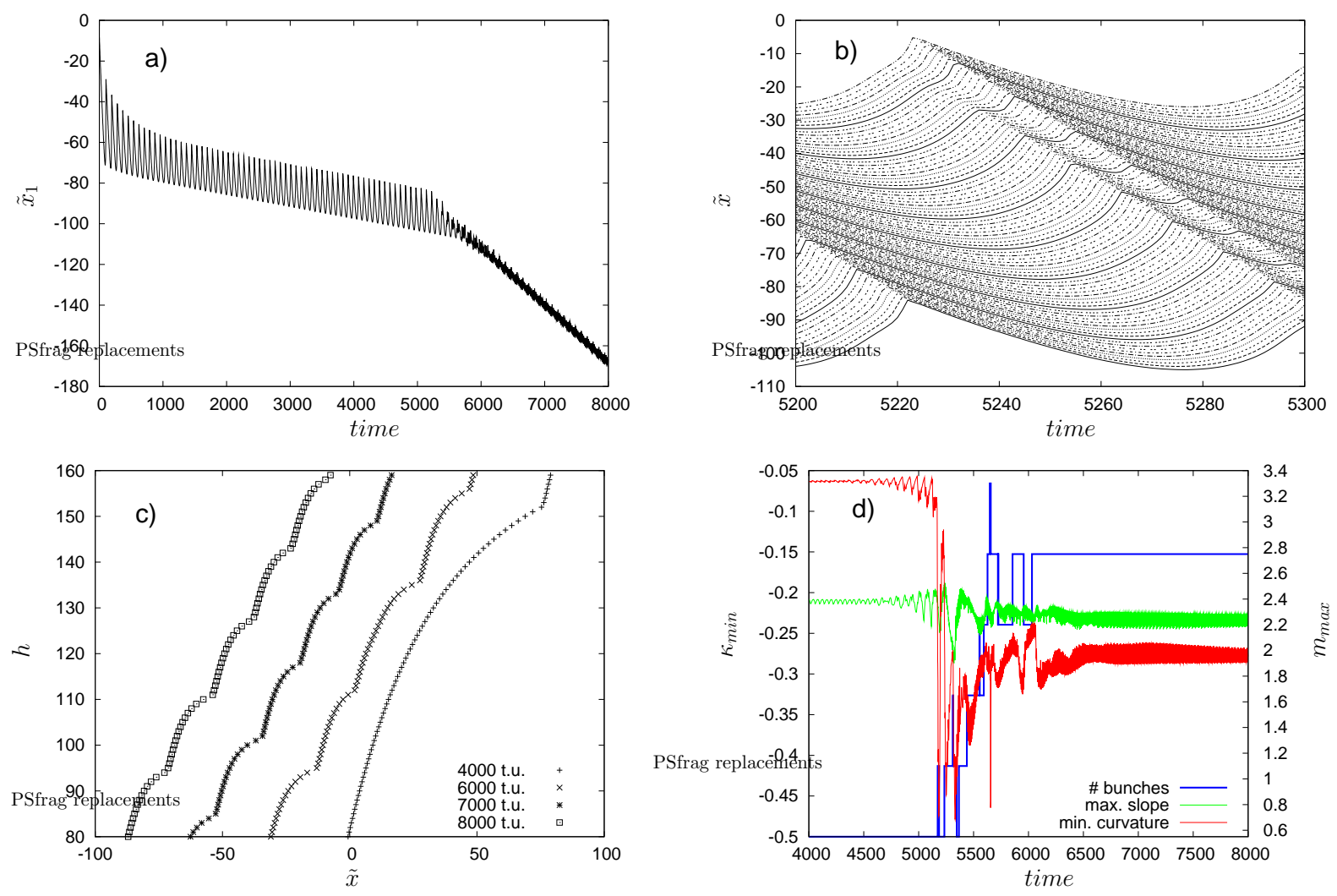

FIG. 8: An example for the splitting of a large bunch in a system of 80 steps with parameters $b=0.7, g=0.05, U=0.05$. a) Time evolution of one of the steps. b) Plot of all step trajectories between 5200t.u. and 5300t.u. c) Comparison of the profiles after 4000 t.u., 6000 t.u., 7000 t.u. and 8000 t.u. d) (Color online) Time evolution of the globally maximal slope, the globally minimal curvature and the number of bunches.

\section{B. Arrested coarsening}

The second type of initial condition is a randomly perturbed equidistant step train. As an example, see Fig. 9, we take $M=40, U=0.2$, a strong perturbation amplitude, an integration time of 8000 t.u., and vary the parameters $g$ and $b$. We use the time evolution of the global maximal slope $m_{\max }(t)$ in order to identify the final state of the simulation. As can be seen in Fig. 9a), after about 2000 t.u. the simulations settled down into a periodic attractor of varying complexity. In Fig. 9b) we show the case of vanishing $g$, which corresponds to a conventional coarsening behavior: After a short time the number of bunches goes to unity and $m_{\max }$ and $\kappa_{\min }$ vary periodically on the characteristic time 

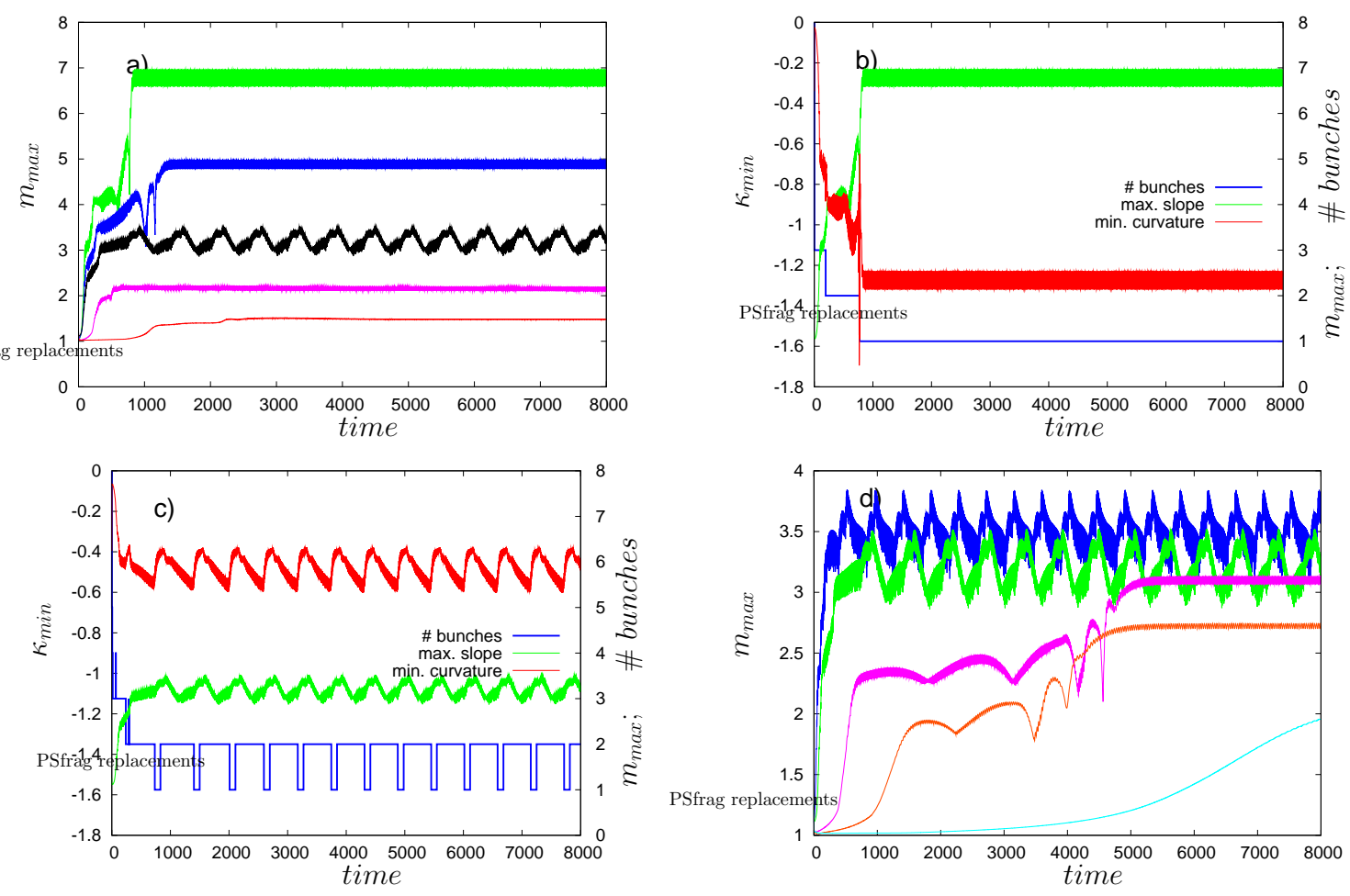

FIG. 9: (Color online) An example of arrested coarsening starting with fluctuating initial conditions of strong amplitude, for $M=40$ and $U=0.2$. a) Maximal slope for $b=0.7$ and (from top to bottom) $g=0,0.01,0.02,0.05,0.09$. b) Number of bunches, maximal slope and minimal curvature for $b=0.7$ and $g=0$. c) As b) for $b=0.7$ and $g=0.02$; d) Maximal slope for $g=0.02$ and (from top to bottom) $b=0.9,0.7,0.4,0.3,0.2$.

scale in which the bunch profile shifts by one average terrace size [19]. By increasing $g$ a point is reached where coarsening into a single bunch no longer occurs, see Fig. 9k). The system is now jumping periodically between configurations with two bunches and one bunch. Upon further increasing $g$ the period of the jumps diverges and the stable state becomes a configuration of two bunches. Finally, Fig. 9d) displays the various regimes of temporal behavior that occur when varying $b$ at fixed $g$.

\section{Phase diagrams}

To somewhat systemize these observations, in Fig. 10 we present two phase diagram where the number of bunches is shown in the $(g, b)$-plane for $b \in[0,1]$ with increments of 

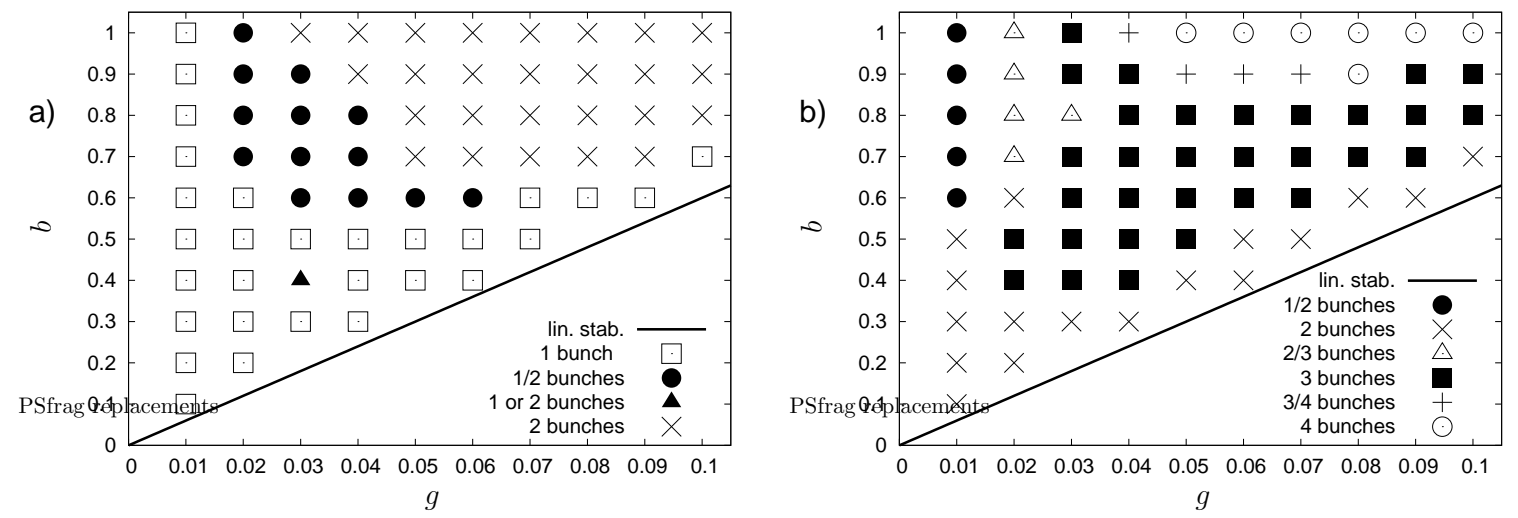

FIG. 10: Stability/instability diagrams showing the number of bunches in the final state, for $M=40$ and different combinations of $b$ and $g$. a) $U=0.2$; b) $U=0.05-\boldsymbol{\Delta}$ : region depending sensitive on the initial condition, $\square$ : 1 bunch, $\bullet$ : $1 / 2$ bunches, $\times$ : 2 bunches, $\triangle: 2 / 3$ bunches, $\mathbf{\square}$ : 3 bunches, $+: 3 / 4$ bunches, $\circ: 4$ bunches, below the line $b=6 g$ : stability

$\triangle b=0.1$ and $g \in[0,0.1]$ with increments of $\triangle g=0.01$. For $U=0.2[$ Fig. [10 a) $]$ the number of bunches in the final stationary state is between one and two. At $g=0.02, b=0.4$ the relaxation time is very sensitive to the initial random configuration and one needs more than 8000 t.u. to switch to the single bunch configuration; moreover, around this point the final state depends sensitively on initial conditions. For $U=0.05$ the number of bunches changes between 4 and an oscillatory state with 1-2 bunches, but a stable state with a single bunch is not seen. In general, we observe that the smaller $U$ the larger is the possible number of bunches. For example for $U=0, M=40, g=0.02$ and $b=0.5$, there are 18 bunches in the final state, corresponding to less than 2.5 steps pro bunch.

In Fig. 11] we show the dependence of the globally maximal slope $m_{\max }$ on $M$ for $g=U=$ 0.04 and $b=0.4$. As we have seen in Fig, $m_{\max }$ generally oscillates in the final state. In order to uniquely define $m_{\max }$ we take for reference its largest value during the last 500 t.u. of the simulation. For a strong initial disturbance $m_{\max }$ grows up to 1.96 at $M=21$ steps and then jumps down to 1.60 at $M=22$, where the step train switches from a single bunch to a configuration with two bunches; for a weak initial disturbance the behavior is similar but the jump occurs slightly earlier. With increasing $M$ this behavior repeats several times until in the region of 80 steps the switching becomes less regular and depends strongly on the initial disturbance. The overall pattern in Fig.11 suggests that the splitting of bunches 


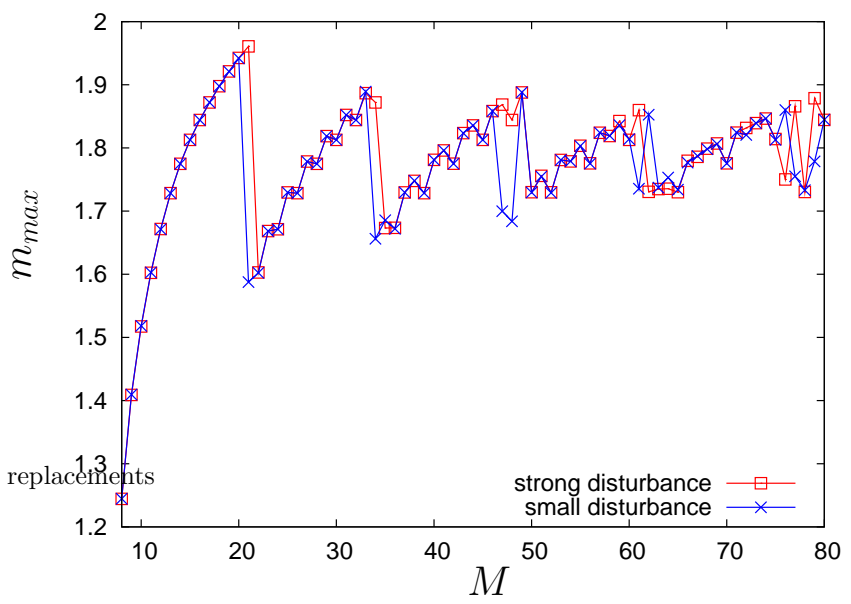

FIG. 11: (Color online) Dependence of the upper boundary of the globally maximal slope $m_{\max }$ on the number of steps $M$ for $g=U=0.04, b=0.4$ and for large and small amplitude of the initial random condition.

is driven by the tendency of the system to keep the maximal slope below a certain limiting value, thus connecting the behavior of the discrete system to the properties of stationary solutions of the continuum equation discussed in Sect IV

\section{SUMMARY AND DISCUSSION}

In this paper we have investigated the extension (7) of a previously studied, minimal model of step bunching during sublimation. Despite the smallness of the dimensionless coefficient $g$ describing the interplay between sublimation and capillarity, the non-conservative nature of the dynamics was found to have profound effects on the linear as well as on the nonlinear level. In order to analytically study the nonlinear behavior of step bunches, we derived and analyzed the continuum evolution equation (10) for the surface profile. Using two different types of approximations, we deduced that the non-conservative terms in this equation place an upper limit on the slope as well as on the wavelength of step bunches. This conclusion was confirmed by a detailed numerical study of the discrete step dynamics, which also revealed additional complex dynamical regimes in which the number of step bunches increases in time or oscillates between different values.

To place our findings into context, we note that a connection between unbounded coarsening of step bunches and the conservative nature of the dynamics has been observed in 
previous work. First, Sato and Uwaha studied step bunching induced by electromigration and observed a saturation of the mean bunch size when sublimation was included [7]. A direct comparison to our results is difficult, however, since they use a different set of step dynamical equations which depend on a larger set of parameters.

Second, the difference between conserved and non-conserved surface dynamics has been addressed in the framework of weakly nonlinear (in the sense of [26]) continuum equations. Working close to the instability threshold, Sato and Uwaha [27] and Misbah and PierreLouis [28] showed that the large-scale dynamics of step bunches in a non-conserved setting is described by the Benney equation. Depending on the size of a term that breaks the leftright symmetry, this equation displays either spatio-temporal chaos or an ordered array of bunches, but no coarsening. On the other hand, the corresponding conservative equation derived in [29] does show unlimited coarsening with the average bunch size growing as $t^{1 / 2}$. In general, deducing the coarsening behavior of a given nonlinear evolution equation by analytic means is a difficult problem, and the methods available so far [30] do not seem to readily carry over to highly nonlinear equations like (10).

\section{Acknowledgements}

We thank P. Politi, B. Ranguelov, S. Stoyanov and V. Tonchev for useful discussions.

\section{Appendix A: Discrete equations for the case of sublimation}

\section{The concentration profile}

In the quasistatic approximation $\partial n_{i} / \partial t=0$ (11) with $F=0$ is a homogeneous ordinary differential equation of second order with the following general solution:

$$
n_{i}(x)=C_{1}^{i} e^{\frac{x}{l_{D}}}+C_{2}^{i} e^{-\frac{x}{l_{D}}}
$$

In order to find the constants of integration $C_{1}^{i}$ and $C_{2}^{i}$ we use the boundary conditions (2):

$$
\begin{aligned}
& \frac{D_{s}}{k_{-}}\left(\frac{C_{1}^{i}}{l_{D}} e^{-\frac{l_{i}}{2 l_{D}}}-\frac{C_{2}^{i}}{l_{D}} e^{+\frac{l_{i}}{2 l_{D}}}\right)=+C_{1}^{i} e^{-\frac{l_{i}}{2 l_{D}}}+C_{2}^{i} e^{+\frac{l_{i}}{2 l_{D}}}-n_{e q}^{0}\left(1+\frac{\mu_{i}}{k T}\right), \\
& \frac{D_{s}}{k_{+}}\left(\frac{C_{1}^{i}}{l_{D}} e^{+\frac{l_{i}}{2 l_{D}}}-\frac{C_{2}^{i}}{l_{D}} e^{-\frac{l_{i}}{2 l_{D}}}\right)=-C_{1}^{i} e^{+\frac{l_{i}}{2 l_{D}}}-C_{2}^{i} e^{-\frac{l_{i}}{2 l_{D}}}+n_{e q}^{0}\left(1+\frac{\mu_{i+1}}{k T}\right) .
\end{aligned}
$$


Substituting $l^{ \pm}:=\frac{D_{s}}{l_{D} k_{ \pm}}=\frac{l_{ \pm}}{l_{D}}$ this becomes

$$
\begin{aligned}
& \left(l^{-}-1\right) C_{1}^{i} e^{-\frac{l_{i}}{2 l_{D}}}-\left(l^{-}+1\right) C_{2}^{i} e^{+\frac{l_{i}}{2 l_{D}}}=-n_{e q}^{0}\left(1+\frac{\mu_{i}}{k T}\right), \\
& \left(l^{+}+1\right) C_{1}^{i} e^{+\frac{l_{i}}{2 l_{D}}}-\left(l^{+}-1\right) C_{2}^{i} e^{-\frac{l_{i}}{2 l_{D}}}=+n_{e q}^{0}\left(1+\frac{\mu_{i+1}}{k T}\right) .
\end{aligned}
$$

After adding both equations and solving for $C_{1}^{i}$ we obtain

$$
C_{1}^{i}=\frac{n_{e q}^{0}\left(\frac{\mu_{i+1}}{k T}-\frac{\mu_{i}}{k T}\right)+C_{2}^{i}\left[\left(l^{-}+1\right) e^{\frac{l_{i}}{2 l_{D}}}+\left(l^{+}-1\right) e^{\left.-\frac{l_{i}}{2 l_{D}}\right]}\right.}{\left(l^{-}-1\right) e^{-\frac{l_{i}}{2 l_{D}}}+\left(l^{+}+1\right) e^{\frac{l_{i}}{2 l_{D}}}} .
$$

We substitute $C_{1}^{i}$ in the first boundary condition:

$$
C_{2}^{i}=\frac{n_{e q}^{0}\left(1+\frac{\mu_{i}}{k T}\right)+C_{1}^{i}\left(l^{-}-1\right) e^{-\lambda_{1} \frac{l_{i}}{2}}}{\left(l^{-}+1\right) e^{-\lambda_{2} \frac{l_{i}}{2}}} .
$$

After back substitution from $C_{2}^{i}$ into $C_{1}^{i}$ and vice versa we get the final form for the constants:

$$
\begin{aligned}
& C_{1}^{i}=\frac{n_{e q}^{0}}{2}\left[\frac{\left(l^{+}-1\right)\left(1+\frac{\mu_{i}}{k T}\right) e^{-\frac{l_{i}}{2 l_{D}}}+\left(l^{-}+1\right)\left(1+\frac{\mu_{i+1}}{k T}\right) e^{\frac{l_{i}}{2 l_{D}}}}{\left(l^{+} l^{-}+1\right) \sinh \frac{l_{i}}{l_{D}}+\left(l^{-}+l^{+}\right) \cosh \frac{l_{i}}{l_{D}}}\right], \\
& C_{2}^{i}=\frac{n_{e q}^{0}}{2}\left[\frac{\left(l^{-}-1\right)\left(1+\frac{\mu_{i}}{k T}\right) e^{-\frac{l_{i}}{2 l_{D}}}+\left(l^{+}+1\right)\left(1+\frac{\mu_{i+1}}{k T}\right) e^{\frac{l_{i}}{2 l_{D}}}}{\left(l^{+} l^{-}+1\right) \sinh \frac{l_{i}}{l_{D}}+\left(l^{-}+l^{+}\right) \cosh \frac{l_{i}}{l_{D}}}\right] .
\end{aligned}
$$

\section{Fluxes and the step velocities}

Using the definition

$$
f_{ \pm}^{i}(x)= \pm \Omega D_{s} \frac{\partial n_{i}(x)}{\partial x} \text { at } x= \pm \frac{l_{i}}{2}
$$

we find for the fluxes to the step bordering the $i$ th terrace of width $l_{i}$ :

$$
\begin{aligned}
& f_{+}^{i}=\frac{\Omega D_{s} n_{e q}^{0}}{l_{D}} \frac{\left(1+\frac{\mu_{i+1}}{k T}\right)\left(l^{-} \sinh \frac{l_{i}}{l_{D}}+\cosh \frac{l_{i}}{l_{D}}\right)-\left(1+\frac{\mu_{i}}{k T}\right)}{\left(l^{+} l^{-}+1\right) \sinh \frac{l_{i}}{l_{D}}+\left(l^{-}+l^{+}\right) \cosh \frac{l_{i}}{l_{D}}}, \text { at step } i+1, \\
& f_{-}^{i}=\frac{\Omega D_{s} n_{e q}^{0}}{l_{D}} \frac{\left(1+\frac{\mu_{i}}{k T}\right)\left(l^{+} \sinh \frac{l_{i}}{l_{D}}+\cosh \frac{l_{i}}{l_{D}}\right)-\left(1+\frac{\mu_{i+1}}{k T}\right)}{\left(l^{+} l^{-}+1\right) \sinh \frac{l_{i}}{l_{D}}+\left(l^{-}+l^{+}\right) \cosh \frac{l_{i}}{l_{D}}}, \text { at step } i .
\end{aligned}
$$

Adding the fluxes from the two neighboring terraces we obtain the expression for the velocity of step $i$ as $v_{i}=\frac{d x_{i}}{d t}=f_{-}^{i}+f_{+}^{i-1}$. 


\section{Linear stability analysis}

Let us assume that $l_{D} \gg l$ and $l_{D} \gg l_{ \pm}$. Then $\sinh \frac{l_{i}}{l_{D}} \approx \frac{l_{i}}{l_{D}}$ and $\cosh \frac{l_{i}}{l_{D}} \approx 1$. For the denominator in the expression for the fluxes (and with $l^{ \pm}=\frac{l_{ \pm}}{l_{D}}$ ) we find

$$
\left(\frac{l_{+} l_{-}}{l_{D}^{2}}+1\right) \sinh \frac{l_{i}}{l_{D}}+\left(\frac{l_{-}}{l_{D}}+\frac{l_{+}}{l_{D}}\right) \cosh \frac{l_{i}}{l_{D}} \approx \frac{l_{-}+l_{+}+l_{i}}{l_{D}},
$$

and the velocity becomes

$$
\begin{aligned}
\frac{1}{\Omega D_{s} n_{e q}^{0}} \frac{d x_{i}}{d t} & \approx \frac{\left(1+\frac{\mu_{i}}{k T}\right)\left(\frac{l_{-} l_{-1}}{l_{D}^{2}}+1\right)-\left(1+\frac{\mu_{i-1}}{k T}\right)}{l_{-}+l_{+}+l_{i-1}}+\frac{\left(1+\frac{\mu_{i}}{k T}\right)\left(\frac{l_{+} l_{i}}{l_{D}^{2}}+1\right)-\left(1+\frac{\mu_{i+1}}{k T}\right)}{l_{-}+l_{+}+l_{i}} \\
& =\frac{\left(1+\frac{\mu_{i}}{k T}\right) \frac{l_{-} l_{i-1}}{l_{D}^{2}}+\left(\frac{\mu_{i}}{k T}-\frac{\mu_{i-1}}{k T}\right)}{l_{-}+l_{+}+l_{i-1}}+\frac{\left(1+\frac{\mu_{i}}{k T}\right) \frac{l_{+} l_{i}}{l_{D}^{2}}+\left(\frac{\mu_{i}}{k T}-\frac{\mu_{i+1}}{k T}\right)}{l_{-}+l_{+}+l_{i}}
\end{aligned}
$$

The chemical potential $\mu_{i}$ can be linearized as

$$
\begin{gathered}
\frac{\mu_{i}}{k T} \approx-g\left[1-3 \frac{\varepsilon_{i+1}-\varepsilon_{i}}{l}-1+3 \frac{\varepsilon_{i}-\varepsilon_{i-1}}{l}\right]=-\frac{3 g}{l}\left(2 \varepsilon_{i}-\varepsilon_{i+1}-\varepsilon_{i-1}\right) \Rightarrow \\
\quad \frac{\mu_{i}}{k T}-\frac{\mu_{i \pm 1}}{k T} \approx-\frac{3 g}{l}\left(-3 \varepsilon_{i \pm 1}+3 \varepsilon_{i}+\varepsilon_{i \pm 2}-\varepsilon_{i \mp 1}\right) \\
\left(1+\frac{\mu_{i}}{k T}\right) \frac{l_{ \pm} l_{i, i-1}}{l_{D}^{2}} \approx \frac{l_{ \pm}}{l_{D}^{2}}\left(l+\varepsilon_{i+1, i}-\varepsilon_{i, i-1}\right)-\frac{3 g l_{ \pm}}{l_{D}^{2}}\left(2 \varepsilon_{i}-\varepsilon_{i+1}-\varepsilon_{i-1}\right) \\
\frac{1}{\Omega D_{s} n_{e q}^{0}} \frac{d x_{i}}{d t} \approx \frac{\left(l_{+}+l_{-}\right) l}{\left(l_{-}+l_{+}+l\right) l_{D}^{2}}+\frac{3 g}{\left(l_{-}+l_{+}+l\right) l}\left(-6 \varepsilon_{i}+4 \varepsilon_{i+1}+4 \varepsilon_{i-1}-\varepsilon_{i+2}-\varepsilon_{i-2}\right) \\
+\frac{l_{-}\left(\varepsilon_{i}-\varepsilon_{i-1}\right)+l_{+}\left(\varepsilon_{i+1}-\varepsilon_{i}\right)}{\left(l_{-}+l_{+}+l\right) l_{D}^{2}} \frac{3 g\left(l_{+}+l_{-}\right)}{\left(l_{-}+l_{+}+l\right) l_{D}^{2}}\left(2 \varepsilon_{i}-\varepsilon_{i+1}+\varepsilon_{i-1}\right) \\
\doteq \frac{1}{\Omega D n_{e q}^{0}}\left(v_{e q}+\frac{d \varepsilon_{i}}{d t}\right)
\end{gathered}
$$

The equation for the perturbation reads

$$
\begin{aligned}
\frac{1}{\Omega D_{s} n_{e q}^{0}} \frac{d \varepsilon_{i}}{d t} & =\frac{3 g}{\left(l_{-}+l_{+}+l\right) l}\left(-6 \varepsilon_{i}+4 \varepsilon_{i+1}+4 \varepsilon_{i-1}-\varepsilon_{i+2}-\varepsilon_{i-2}\right) \\
& +\frac{l_{-}\left(\varepsilon_{i}-\varepsilon_{i-1}\right)+l_{+}\left(\varepsilon_{i+1}-\varepsilon_{i}\right)}{\left(l_{-}+l_{+}+l\right) l_{D}^{2}}-\frac{3 g\left(l_{+}+l_{-}\right)}{\left(l_{-}+l_{+}+l\right) l_{D}^{2}}\left(2 \varepsilon_{i}-\varepsilon_{i+1}+\varepsilon_{i-1}\right)
\end{aligned}
$$

Using the Fourier expression $\varepsilon=\varepsilon_{0} e^{i k n+\omega(k) t}$ we find the dispersion relation $\omega(k)$ :

$$
\begin{aligned}
\frac{\omega(k)}{\Omega D_{s} n_{e q}^{0}} & =\frac{3 g}{\left(l_{-}+l_{+}+l\right) l}\left[-6+4\left(e^{i k}+e^{-i k}\right)-\left(e^{i 2 k}+e^{-i 2 k}\right)\right] \\
& +\frac{l_{-}\left(1-e^{-i k}\right)+l_{+}\left(e^{i k}-1\right)}{\left(l_{-}+l_{+}+l\right) l_{D}^{2}}-\frac{3 g\left(l_{+}+l_{-}\right)}{\left(l_{-}+l_{+}+l\right) l_{D}^{2}}\left(2-\left(e^{i k}+e^{-i k}\right)\right) .
\end{aligned}
$$


For small $k$ we can expand the exponential functions as

$$
\begin{aligned}
2-\left(e^{i k}+e^{-i k}\right) & =2(1-\cos k) \approx 2\left(1-1+\frac{k^{2}}{2}\right)=k^{2}, \\
6-4\left(e^{i k}+e^{-i k}\right)+\left(e^{i 2 k}+e^{-i 2 k}\right) & =4(\cos k-1)^{2} \approx k^{4}, \\
1-e^{-i k} & \approx 1-\left(1-i k+\frac{k^{2}}{2}\right)=i k+\frac{k^{2}}{2},
\end{aligned}
$$

which yields an expression for the real part $R e[\omega]$ :

$$
R e[\omega]=\frac{\Omega D_{s} n_{e q}^{0}}{l_{D}^{2}} \frac{\left(l_{-}+l_{+}\right)}{\left(l_{-}+l_{+}+l\right)}\left[\frac{\left(l_{-}-l_{+}\right)}{2}-3 g\right] k^{2}-\frac{\Omega D_{s} n_{e q}^{0} 3 g}{\left(l_{-}+l_{+}+l\right) l} k^{4} .
$$

Further, if $l_{ \pm} \gg l$

$$
R e[\omega]=\frac{\Omega D_{s} n_{e q}^{0}}{l_{D}^{2}}\left[\frac{\left(k_{+}-k_{-}\right)}{2\left(k_{+}+k_{-}\right)}-3 g\right] k^{2}-3 g \frac{\Omega D_{s} n_{e q}^{0}}{l} \frac{k_{-} k_{+}}{k_{-}+k_{+}} k^{4} .
$$

For instability $R e[\omega]$ has to be positive. The prefactor $A_{4}$ of the $k^{4}$-term is always negative

and thus acts as a relaxation term. For $k$ very small the linear instability condition $b>6 g$ $\left(A_{2}>0\right)$ follows.

\section{Discrete equations in the limit $l_{D} \gg l_{ \pm} \gg l_{i}$}

From equation (A6) with $l_{ \pm} \gg l_{i}$ we get

$$
\begin{aligned}
\frac{d x_{i}}{d t} & \approx \frac{\Omega D_{s} n_{e q}^{0}}{l_{-}+l_{+}}\left(1+\frac{\mu_{i}}{k T}\right)\left(\frac{l_{-} l_{i-1}}{l_{D}^{2}}+\frac{l_{+} l_{i}}{l_{D}^{2}}\right)+\frac{\Omega D_{s} n_{e q}^{0}}{l_{-}+l_{+}}\left(2 \frac{\mu_{i}}{k T}-\frac{\mu_{i-1}}{k T}-\frac{\mu_{i+1}}{k T}\right) \\
& =\left(1+g \nu_{i}\right) R_{e}\left(\frac{l_{-} l_{i-1}+l_{+} l_{i}}{l_{-}+l_{+}}\right)+U R_{e}\left(2 \nu_{i}-\nu_{i-1}-\nu_{i+1}\right) \\
& =\left(1+g \nu_{i}\right) R_{e}\left(\frac{1+b}{2} l_{i-1}+\frac{1-b}{2} l_{i}\right)+U R_{e}\left(2 \nu_{i}-\nu_{i-1}-\nu_{i+i}\right)
\end{aligned}
$$

\section{Appendix B: Continuum limit}

Similar to [20], we treat the part of (7) without the $U$-term first,

$$
\frac{d x_{n}}{d t}=\left(1+g \nu_{n}\right)\left(\frac{1-b}{2} l_{n}+\frac{1+b}{2} l_{n-1}\right)
$$


where $l_{n}=x_{n+1}-x_{n}$. To obtain the continuum limit of $\left(\frac{1-b}{2} l_{n}+\frac{1+b}{2} l_{n-1}\right)$, we rewrite it in the form

$$
\begin{aligned}
\frac{d x_{n}}{d t} & =\left(1+g \nu_{n}\right)\left(\frac{l_{n}+l_{n-1}}{2}-\frac{b}{2}\left(l_{n}-l_{n-1}\right)\right) \\
\frac{d x_{n+1}}{d t} & =\left(1+g \nu_{n+1}\right)\left(\frac{l_{n+1}+l_{n}}{2}-\frac{b}{2}\left(l_{n+1}-l_{n}\right)\right)
\end{aligned}
$$

Substracting,

$$
\begin{aligned}
\frac{d l_{n}}{d t} & =\frac{l_{n+1}-l_{n-1}}{2}-\frac{b}{2}\left(l_{n+1}-2 l_{n}+l_{n-1}\right) \\
& +g \frac{\nu_{n+1} l_{n+1}-\nu_{n} l_{n}}{2}+g \frac{\nu_{n+1} l_{n}-\nu_{n} l_{n-1}}{2}-g b \frac{\nu_{n+1} l_{n+1}+\nu_{n} l_{n-1}-\left(\nu_{n+1}+\nu_{n}\right) l_{n}}{2}
\end{aligned}
$$

Let us treat the $g$-independent term first. Performing the Fourier transform $l_{n}=\sum_{q} e^{i q n} l_{q}$, we obtain

$$
\sum_{q} e^{i q n} \frac{d l_{q}}{d t}=\sum_{q} e^{i q n}\left[\frac{1}{2}\left(e^{i q}-e^{-i q}\right)-b \frac{e^{i q}+e^{-i q}-2}{2}\right] l_{q}
$$

Expanding for small $q$,

$$
\sum_{q} e^{i q n} \frac{d l_{q}}{d t} \approx \sum_{q} e^{i q n}\left[\left((i q)+\frac{(i q)^{3}}{3 !}+\ldots\right)-b\left(\frac{(i q)^{2}}{2 !}+\frac{(i q)^{4}}{4 !}\right)\right] l_{q}
$$

Noting that $(i q) \sum_{q} e^{i q n} l_{q}=\frac{\partial}{\partial n}\left(\sum_{q} e^{i q n} l_{q}\right)=\frac{\partial}{\partial n} l_{n}$ etc., one can rewrite the previous equation as

$$
\frac{d l_{n}}{d t}=\left[\left(\frac{\partial}{\partial n}+\frac{1}{3 !} \frac{\partial^{3}}{\partial n^{3}}\right)-b\left(\frac{1}{2 !} \frac{\partial^{2}}{\partial n^{2}}+\frac{1}{4 !} \frac{\partial^{4}}{\partial n^{4}}\right)\right] l_{n} .
$$

Now, we can use $\frac{\partial}{\partial n}=h_{0} \frac{\partial}{\partial h}=\frac{\partial}{\partial h}$ (note that $\left.h_{0}=1\right)$ and $l_{n} \approx 1 / m(x)=1 /(\partial h / \partial x)=\partial x / \partial h$. We obtain

$$
\frac{\partial}{\partial h} \frac{d x}{d t}=\frac{\partial}{\partial h}\left[\left(1+\frac{1}{3 !} \frac{\partial^{2}}{\partial h^{2}}\right)-b\left(\frac{1}{2 !} \frac{\partial}{\partial h}+\frac{1}{4 !} \frac{\partial^{3}}{\partial h^{3}}\right)\right] \Delta(x)
$$

where $\Delta(x)=1 / m(x)$. Integrating this equation we find

$$
\frac{d x}{d t}=\left[\left(1+\frac{1}{3 !} \frac{\partial^{2}}{\partial h^{2}}\right)-b\left(\frac{1}{2 !} \frac{\partial}{\partial h}+\frac{1}{4 !} \frac{\partial^{3}}{\partial h^{3}}\right)\right] \frac{\partial x(t)}{\partial h} .
$$

Inserting the terms proportional to $g$ and approximating

$$
\nu_{n}=\left(\frac{l}{l_{n-1}}\right)^{3}-\left(\frac{l}{l_{n}}\right)^{3} \approx-l_{n} \frac{\partial}{\partial x}\left(\frac{l}{\Delta}\right)^{3}=-\frac{1}{m}\left(m^{3}\right)^{\prime}=-\frac{3}{2}\left(m^{2}\right)^{\prime}
$$

we get

$$
\frac{d x}{d t}=\left(1-\frac{3 g}{2}\left(m^{2}\right)^{\prime}\right)\left[\left(1+\frac{1}{3 !} \frac{\partial^{2}}{\partial h^{2}}\right)-b\left(\frac{1}{2 !} \frac{\partial}{\partial h}+\frac{1}{4 !} \frac{\partial^{3}}{\partial h^{3}}\right)\right] \Delta(x, t)
$$


The next important step is to carry out the Lagrange transformation [20, 31] from $x(n, t)=x\left(h=n h_{0}, t\right)=x(h, t)$ to $h(x, t)$. First, note that $\frac{d x}{d t}=-\left(\frac{d x}{d h}\right) \frac{d h}{d t}=-\Delta \frac{d h}{d t}$. Substituting this and using $\frac{\partial}{\partial h}=\left(\frac{d x}{d h}\right) \frac{\partial}{\partial x}=\Delta \frac{\partial}{\partial x}$,

$$
-\frac{d h}{d t}=\left(1-\frac{3 g}{2}\left(m^{2}\right)^{\prime}\right)+\left(1-\frac{3 g}{2}\left(m^{2}\right)^{\prime}\right) \frac{\partial}{\partial x}\left[\left(\frac{1}{3 !} \frac{\partial}{\partial h}\right)-b\left(\frac{1}{2 !}+\frac{1}{4 !} \frac{\partial^{2}}{\partial h^{2}}\right)\right] \Delta(x, t)
$$

or

$$
\begin{aligned}
-1=\frac{d h}{d t} & +\frac{\partial}{\partial x}\left\{-\frac{3 g}{2}\left(m^{2}\right)+\left[\left(\frac{1}{3 !} \frac{\partial \Delta}{\partial h}\right)-b\left(\frac{\Delta}{2 !}+\frac{1}{4 !} \frac{\partial^{2} \Delta}{\partial h^{2}}\right)\right]\right\} \\
- & \frac{3 g}{2}\left(m^{2}\right)^{\prime} \frac{\partial}{\partial x}\left[\left(\frac{1}{3 !} \frac{\partial \Delta}{\partial h}\right)-b\left(\frac{\Delta}{2 !}+\frac{1}{4 !} \frac{\partial^{2} \Delta}{\partial h^{2}}\right)\right]
\end{aligned}
$$

Now we estimate the term in the square brackets, using $\Delta=1 / m, \frac{\partial}{\partial h}=\Delta \frac{\partial}{\partial x}=(1 / m) \frac{\partial}{\partial x}$

$$
\left[\left(\frac{1}{3 !} \frac{\partial \Delta}{\partial h}\right)-b\left(\frac{\Delta}{2 !}+\frac{1}{4 !} \frac{\partial^{2} \Delta}{\partial h^{2}}\right)\right]=\frac{1}{6 m} \frac{\partial}{\partial x}\left(\frac{1}{m}\right)-\frac{b}{2 m}-\frac{b}{24} \frac{\partial^{2} \Delta}{\partial h^{2}}
$$

Aiming at smooth solutions, we ignore high order derivatives, approximating

$$
[\ldots] \approx-\frac{b}{2 m}-\frac{m^{\prime}}{6 m^{3}}
$$

Substituting we obtain

$$
\frac{d h}{d t}+\frac{\partial}{\partial x}\left(-\frac{3 g}{2}\left(m^{2}\right)-\frac{b}{2 m}-\frac{m^{\prime}}{6 m^{3}}\right)=-1+\frac{3 g}{2}\left(m^{2}\right)^{\prime}\left[-\frac{b}{2 m}-\frac{m^{\prime}}{6 m^{3}}\right]^{\prime}
$$

The term proportional to $U$ gives a contribution to the full derivative,

$$
\frac{d h}{d t}+\frac{\partial}{\partial x}\left(-\frac{3 g}{2}\left(m^{2}\right)-\frac{b}{2 m}-\frac{m^{\prime}}{6 m^{3}}+\frac{3 U}{2} \frac{\left(m^{2}\right)^{\prime \prime}}{m}\right)=-1+\frac{3 g}{2}\left(m^{2}\right)^{\prime}\left[-\frac{b}{2 m}-\frac{m^{\prime}}{6 m^{3}}\right]^{\prime}
$$

The terms proportional to $g$ on the RHS break the conservative character of the equation, and are important for the dynamics as discussed in the main text. However, our numerical data (not shown) suggest that the best (though not perfect) agreement with the discrete system is obtained by keeping only the nonconservative term of the lowest order,

$$
\frac{d h}{d t}+\frac{\partial}{\partial x}\left(-\frac{3 g}{2}\left(m^{2}\right)-\frac{b}{2 m}-\frac{m^{\prime}}{6 m^{3}}+\frac{3 U}{2} \frac{\left(m^{2}\right)^{\prime \prime}}{m}\right)=-1+\frac{3 g}{2}\left(m^{2}\right)^{\prime}\left[-\frac{b}{2 m}\right]^{\prime}
$$

Expanding the derivative on the RHS, we obtain (10).

[1] H.-C. Jeong and E.D. Williams, Surf. Sci. Rep. 34 (1999). 
[2] J. Krug, in Multiscale Modeling of Epitaxial Growth, Int. Ser. Num. Math. 149, ed. by A. Voigt (Birkhäuser, Basel 2005), p.69.

[3] O. Pierre-Louis, C.R. Physique 6, 11 (2005).

[4] J. Krug, in Nonlinear Dynamics of Nanosystems, ed. by G. Radons, B. Rumpf and H.G. Schuster (Wiley, Weinheim 2010), p.143.

[5] W.K. Burton, N. Cabrera, and F.C. Frank, Phil. Trans. R. Soc. London Ser. A 243, 299 (1951).

[6] S. Stoyanov, Jpn. J. Appl. Phys., Part 1 30, 1 (1991).

[7] M. Sato and M. Uwaha, Surf. Sci. 442, 318 (1999).

[8] J. Tersoff, Y.H. Phang, Z. Zhang and M.G. Lagally, Phys. Rev. Lett. 75, 2730 (1995).

[9] R.L. Schwoebel, J. Appl. Phys. 40, 614 (1969).

[10] A. Pimpinelli, I. Elkinani, A. Karma, C. Misbah and J. Villain, J. Phys.: Condens. Matter 6, 2661 (1994).

[11] M. Uwaha, Y. Saito, and M. Sato, J. Cryst. Growth 146, 164 (1995).

[12] M. Sato and M. Uwaha, Surf. Sci. 493, 494 (2001).

[13] M.H. Xie, S.Y. Leung, and S.Y. Tong, Surf. Sci. 515, L459 (2002).

[14] F. Slanina, J. Krug, and M. Kotrla, Phys. Rev. E 71, 041605 (2005).

[15] O. Pierre-Louis, Surf. Sci. 529, 114 (2003).

[16] P.-W. Fok, R.R. Rosales, and D. Margetis, Phys. Rev. B 76, 033408 (2007).

[17] J. Krug, Adv. Phys. 46, 139 (1997).

[18] J. Krug, V. Tonchev, S. Stoyanov, and A. Pimpinelli, Phys. Rev. B 71, 045412 (2005)

[19] V. Popkov and J. Krug, Europhys. Lett., 72(6), 1025 (2005).

[20] J. Krug, in Dynamics of Fluctuating Interfaces and Related Phenomena edited by D. Kim et al. (World Scientific, Singapore, 1997), p.95.

[21] E.E. Gruber and W.W. Mullins, J. Phys. Chem. Solids, 28, 875 (1967).

[22] M. Giesen, Prog. Surf. Sci. 68 (2001).

[23] D-J. Liu and J.D. Weeks, Phys. Rev. B 57, 14891 (1998).

[24] V. Popkov and J. Krug, Phys. Rev. B 73, 235430 (2006).

[25] W.H. Press, S.A. Teukolsky, W.T. Vetterling, and B.P. Flannery, Numerical Recipes in C, Second Edition (Cambridge University Press, 1992).

[26] O. Pierre-Louis, Europhys. Lett. 72, 894 (2005). 
[27] M. Sato and M. Uwaha, Europhys. Lett. 32, 639 (1995).

[28] C. Misbah and O. Pierre-Louis, Phys. Rev. E 53, R4318 (1996).

[29] F. Gillet, Z. Csahok and C. Misbah, Phys. Rev. B 63, 241401(R) (2001).

[30] P. Politi and C. Misbah, Phys. Rev. E 73, 036133 (2006).

[31] J. Krug, J. Stat. Phys. 87, 505 (1997). 